\title{
The effects of posterior continuous curvilinear capsulorhexis on contrast sensitivity
}

E Bozkurt, AT Yazıcı, G Pekel, E Pekel,

Ş Bayraktar and ÖF Yılmaz

\begin{abstract}
Purpose We aimed at evaluating the effects of posterior continuous curvilinear capsulorhexis (PCCC) on contrast sensitivity.

Materials and methods In this prospective, randomized, bilateral clinical study, 40 eyes of 20 patients who underwent bilateral cataract surgery were included. The phacoemulsification machine, viscoelastic substances, surgical tools, and intraocular lenses (IOLs) were the same for both eyes. The only difference was that we conducted the PCCC procedure before IOL implantation to one of the eyes of the patients. The selection of the eye that underwent the PCCC procedure was decided randomly.
\end{abstract}

Results The mean photopic contrast sensitivity values at spatial frequencies of 1.5, $3,6,12$, and $18 \mathrm{cpd}$ (cycles per degree) were $41.55,59.90,61.25,32.35$, and 9.75, respectively, and for the control group these values were $39.05,56.60,57.95,29.80$, and 8.75 , respectively. The mean mesopic contrast sensitivity values at special frequencies of 1.5, 3, 6, 12, and $18 \mathrm{cpd}$ were $41.20,54.75,55.55,31.70$, and 9.00 , respectively, and for the control group these values were $38.35,51.70,52.15,30.05$, and 8.00 , respectively. The mean contrast sensitivity values of the eyes that underwent the PCCC procedure were slightly better than the fellow eyes at all spatial frequencies, but the difference was statistically insignificant $(P>0.05)$.

Conclusions In early post-operative period, the PCCC procedure exerts some positive effects on contrast sensitivity although these effects are statistically insignificant.

Eye (2010) 24, 805-809; doi:10.1038/eye.2009.224; published online 4 September 2009

Keywords: posterior continuous curvilinear capsulorhexis; contrast sensitivity; phacoemulsification

\section{Introduction}

Cataract surgery has developed rapidly over the past decades. Clinical experience has led to the insight that Snellen visual acuity is inadequate as a sole parameter for describing the quality-of-vision outcomes of cataract surgery. Contrast sensitivity testing will also will have a role in determining the quality of vision. ${ }^{1-4}$ Visual acuity may be normal in the presence of reduced contrast sensitivity. There are many patients whose subjective complaints do not correspond with their apparent vision in the examining room. Descriptions of difficulty in recognizing faces or functioning at low light levels are common. ${ }^{5}$

Contrast sensitivity measurements at different spatial frequencies, yielding the so-called contrast sensitivity function, evaluate visual function more comprehensively than does Snellen visual acuity, because the latter indicates only the maximal resolution of the eye for high-contrast stimuli.

Cataract extraction with intraocular lens (IOL) implantation is the most frequently performed surgical procedure in the world. The most common complication is posterior capsule opacification (PCO), which results in decreased visual performance. PCO complication is related to some factors such as follow-up time after cataract surgery, type of IOL implanted, surgical skill and technique, as well as the ocular and systemic history of the patient. ${ }^{6-8}$

The primary treatment option for PCO is Nd:YAG laser capsulotomy. Posterior continuous curvilinear capsulorhexis (PCCC) is also performed for preventing PCO after cataract surgery. PCCC is a well-known and well-documented procedure in the literature., In this study, we aimed at detecting the effects of PCCC on contrast sensitivity. We evaluated the contrast sensitivity function
Department of

Ophthalmology, Beyoglu Eye Research and Training Hospital, Istanbul, Turkey

Correspondence: G Pekel, Department of

Ophthalmology, Beyoglu Eye Research and Training Hospital, Kuledibi, Beyoglu, Istanbul, Avrupa yakasi 34020, Turkey. Tel: + 090505855 9704; Fax: + 0902122526109. E-mail: gkhanpekel@ yahoo.com

Received: 20 April 2009 Accepted in revised form: 15 July 2009;

Published online:

4 September 2009 
under photopic and mesopic conditions, in the absence of a glare source.

\section{Materials and methods}

In all, 48 eyes of 24 patients (12 males and 12 females) who underwent bilateral cataract surgery were included in this prospective interventional case series. All of the operations were performed between December 2007 and July 2008. For all of the experiments, informed consent was obtained from all subjects after the nature of the study had been explained fully. Patients with bilateral visually significant senile cataract, corneal astigmatism $<1.50 \mathrm{D}$ and post-operative best corrected visual acuity (BCVA) of $20 / 25$ or better (Snellen chart) were eligible for inclusion in the study. Exclusion criteria were any ocular diseases, such as corneal opacities or irregularity, dry eye, amblyopia, anisometropia, glaucoma, retinal abnormalities, surgical complications, IOL tilt, IOL decentralization, any neurological problem, diabetes mellitus, taking medication that might affect contrast sensitivity, insufficient mental ability to perform the tests, and any physical disability that might make it difficult to perform the test or follow-up. Four patients were excluded from the study because three of them did not meet the criteria of BCVA $>20 / 25$ and one of them had different IOL types in his right and left eyes. After this screening process, 40 eyes of 20 patients (10 males and 10 females) were included in the study. The IOL power ranged from 19.50 to $24.50 \mathrm{D}$.

All surgeries were performed by one experienced surgeon (EB) using the same three-step clear corneal incision and four-quadrant divide and conquer technique. Anterior continuous curvilinear capsulorexis with an approximate diameter of $5.0 \mathrm{~mm}$ and PCCC with an approximate diameter of $3.0 \mathrm{~mm}$ were created. The IOLs were implanted in the capsular bag. The phacoemulsification machine, viscoelastic substances, surgical tools, and IOLs were the same for both eyes. Three-piece foldable aspheric hydrophilic acrylic IOLs were implanted in all operations. The only difference was that we performed the PCCC procedure before IOL implantation to one of the eyes of the patients. The selection of the eye that underwent the PCCC procedure was decided randomly. Cataract surgeries were performed sequentially, and the time gap between the operations of both eyes was $\sim 1$ month.

Functional acuity contrast testing (F.A.C.T.) ${ }^{11}$ was measured between 2 and 4 months after surgery because of the possibility of post-operative cystoid macular oedema (CME), using the Optec 6500 vision testing system (Stereo Optical Co. Inc., Chicago, IL, USA) with best spectacle correction and natural pupil under both the photopic condition (target luminance value of
$85 \mathrm{~cd} / \mathrm{m}^{2}$ ) and mesopic condition (target luminance value of $3 \mathrm{~cd} / \mathrm{m}^{2}$ ).

Contrast sensitivity values were converted to numerical values by using a conversion chart of F.A.C.T. Contrast sensitivity values were compared at all five spatial frequencies $(1.5,3,6,12$, and $18 \mathrm{cpd}$ (cycles per degree)). For statistical analysis, SPSS 14.0 software for Windows (SPSS Inc., Chicago, IL, USA) was used to summarize baseline characteristics and outcomes. $P$-values $<0.05$ were considered to be statistically significant.

\section{Results}

In all, 40 eyes of 20 patients were enrolled in this study. This study comprised 10 male (50\%) and 10 female (50\%) patients. Their ages ranged from 51 to 87 years. The mean age was 66.25 (SD: 10.59) years. All the eyes that were examined in this study had a mean post-operative BCVA of $20 / 25$ or better. The mean topographic astigmatism in the PCCC group and the control group was $0.66 \pm 0.32$ and $0.72 \pm 0.34 \mathrm{D}(P=0.58)$, respectively. The IOL power was estimated at $19.50 \pm 24.50 \mathrm{D}$ and there was no significant difference between the mean IOL powers of the two groups $(21.70 \pm 1.36 \mathrm{D}$ for the PCCC group and $21.62 \pm 1.22 \mathrm{D}$ for the control group; $P=0.63$ ). We did not encounter any complications intra- and post-operatively.

At all spatial frequencies (1.5, 3, 6, 12, $18 \mathrm{cpd})$, under both day (photopic) and night (mesopic) conditions, the contrast sensitivity values of the eyes that underwent PCCC were slightly greater than those of the other eyes. However, all of the differences between the values of the two groups were statistically insignificant. Figures 1 and 2 show contrast sensitivity curves. Tables 1 and 2 show numerical contrast sensitivity values and $P$-values under photopic and mesopic conditions.

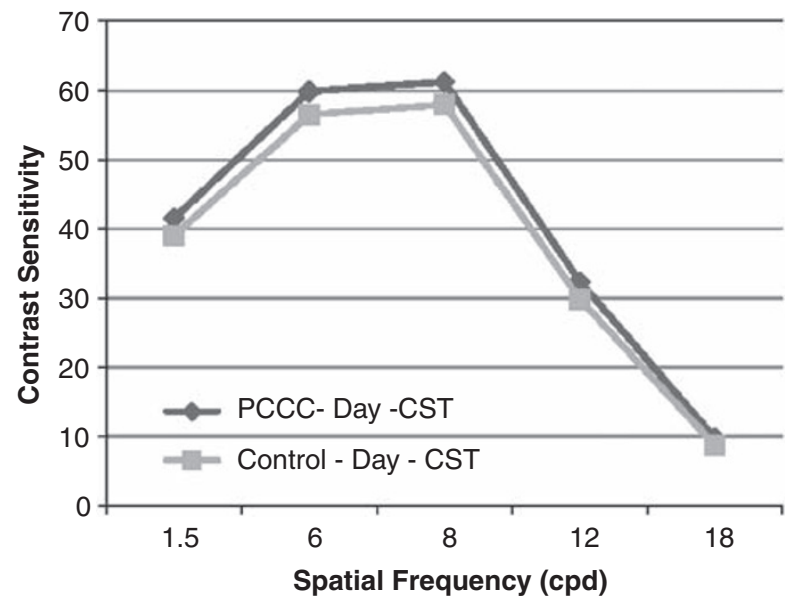

Figure 1 Photopic contrast sensitivity curves of the eyes that underwent posterior continuous curvilinear capsulorhexis (PCCC) and the fellow eyes. (CST: contrast sensitivity test, cpd: cycles per degree) 


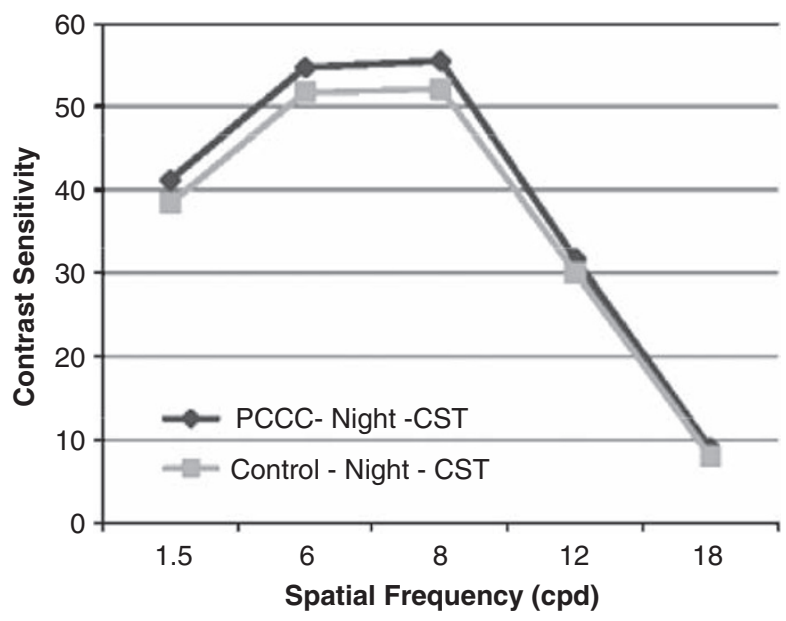

Figure 2 Mesopic contrast sensitivity curves of the eyes that underwent posterior continuous curvilinear capsulorhexis (PCCC) and the fellow eyes. (CST: contrast sensitivity test, cpd: cycles per degree)

Table 1 Photopic contrast sensitivity values of the eyes that underwent posterior continuous curvilinear capsulorhexis (PCCC) and the fellow eyes

\begin{tabular}{lccc}
\hline $\begin{array}{l}\text { Spatial frequency } \\
\text { (cpd) }\end{array}$ & $\begin{array}{c}\text { PCCC } \\
\text { (mean (SD)) }\end{array}$ & $\begin{array}{c}\text { Control } \\
\text { (mean }(S D))\end{array}$ & P-value \\
\hline 1.5 & $41.55(10.82)$ & $39.05(11.48)$ & 0.37 \\
3.0 & $59.90(11.53)$ & $56.50(19.39)$ & 0.36 \\
6.0 & $61.25(15.22)$ & $57.95(24.93)$ & 0.40 \\
12 & $32.35(10.31)$ & $29.80(11.41)$ & 0.39 \\
18 & $9.75(3.11)$ & $8.75(3.51)$ & 0.16 \\
\hline
\end{tabular}

Abbreviation: cpd, cycles per degree.

Table 2 Mesopic contrast sensitivity values of the eyes that underwent posterior continuous curvilinear capsulorhexis (PCCC) and the fellow eyes

\begin{tabular}{lccc}
\hline $\begin{array}{l}\text { Spatial frequency } \\
\text { (cpd) }\end{array}$ & $\begin{array}{c}\text { PCCC } \\
\text { (mean (SD)) }\end{array}$ & $\begin{array}{c}\text { Control } \\
\text { (mean }(S D))\end{array}$ & P-value \\
\hline 1.5 & $41.20(12.43)$ & $38.35(11.20)$ & 0.40 \\
3.0 & $54.75(16.03)$ & $51.70(20.41)$ & 0.25 \\
6.0 & $55.55(20.31)$ & $52.15(20.20)$ & 0.35 \\
12 & $31.70(10.01)$ & $30.05(12.52)$ & 0.44 \\
18 & $9.00(2.87)$ & $8.00(2.51)$ & 0.09 \\
\hline
\end{tabular}

Abbreviation: cpd, cycles per degree.

\section{Discussion}

In this prospective randomized trial, the impact of posterior curvilinear capsulorhexis (PCCC) on contrast sensitivity was evaluated. In primary PCCC, the central part of the posterior capsule is removed during cataract surgery to prevent equatorial lens epithelial cell migration onto the posterior capsule from reaching the visual axis. PCCC is used frequently to prevent more severe PCO formation in children's eyes in combination with anterior vitrectomy or posterior optic capture. ${ }^{12,13}$ However, PCCC is not a standard procedure for the surgical treatment of age-related cataracts.

Despite the developments in IOL design and improvements in surgical techniques, $\mathrm{PCO}$ is still the most common complication of cataract surgery. ${ }^{14}$ The effect of PCO on BCVA, contrast sensitivity, and glare sensitivity in otherwise healthy eyes has been established. ${ }^{15,16} \mathrm{Nd}$ :YAG laser capsulotomy is the main treatment option for PCO.

In our study, we measured contrast sensitivity values of the subjects in 2-4 months after the operations; therefore, we had the chance to measure the fellow eyes that had not undergone the PCCC procedure in a PCOfree situation. In this way, we also attempted to evaluate the pure effect of clear posterior capsule that was free of PCO on contrast sensitivity. Recently, some investigators developed software programmes to measure the severity of PCO using analysis of retro illumination images; but we assessed PCO at the slit lamp from reflected and backward scattered light. ${ }^{17,18}$

In this study, at all spatial frequencies, under both day and night conditions, contrast sensitivity values of the eyes that underwent PCCC were slightly greater than those of the fellow eyes. However, all of the differences between the values of the two groups were statistically insignificant $(P>0.05)$. Although this result was statistically insignificant, it is important to remember that contrast sensitivity of the fellow eyes was measured in cases of clear posterior capsule; therefore, it is obvious that as PCO formation occurs with the passage of time, the result may become a statistically significant value. In addition, although it was a subjective evaluation, most of the patients (13 patients, 65\%) reported that the visual quality was better in their eyes after undergoing PCCC.

Recent studies have shown that PCO significantly disturbs contrast sensitivity in addition to visual acuity and these visual functions improve significantly after $\mathrm{Nd}$ :YAG laser posterior capsulotomy. Most of these studies reported a correlation between the improvement of visual acuity and contrast sensitivity after Nd:YAG laser capsulotomy. ${ }^{19,20}$ Hayashi et al, ${ }^{16}$ noted specifically that visual acuity has a stronger association with PCO than does contrast sensitivity or glare sensitivity and also reported that in patients who had a visual acuity of 20/25 or better, visual acuity improved significantly after capsulotomy, but contrast sensitivity did not show significant improvement. However, Tan $e t a^{21}$ implied that the improvement in contrast sensitivity was associated poorly with that in visual acuity and that 
contrast sensitivity testing is more sensitive than is visual acuity in the early stages of PCO.

Vock et $a l^{22}$ had examined the effect of PCCC on clinical performance of a hydrophilic acrylic IOL, and similar to our results they found that neither BCVA nor contrast sensitivity were significantly different between the PCCC group and the non-PCCC group; but in contrast to our study, they had applied Nd:YAG laser capsulotomy to some patients in the non-PCCC group and their study had observed contrast sensitivity values at a late post-operative period. Mela $e t a l^{23}$ compared contrast sensitivity in patients with intact posterior capsules having posterior chamber monofocal IOL and in patients with ruptured posterior capsules having anterior chamber monofocal IOL, and found an impairment of contrast sensitivity at intermediate and high spatial frequencies with an intact posterior capsule.

As both PCCC and Nd:YAG laser capsulotomy give similar results theoretically and clinically in terms of visual acuity and contrast sensitivity improvements, it is logical to compare all the other aspects of these two procedures. In the PCCC procedure, the diameter of the rhexis is usually standard and is $\sim 3 \mathrm{~mm}$; it is very hard to enlarge the rhexis after the operation, whereas in Nd:YAG laser posterior capsulotomy, it is easy to modify the shape and width of the capsulotomy in different sessions. However, it was reported that visual functions, including contrast sensitivity, did not have a significant correlation with the area of the capsulotomy opening and even relatively small capsulotomy openings may be sufficient to improve visual function. ${ }^{16}$

Neodymium:YAG laser capsulotomy can result in significant morbidity from post-operative complications. These include IOL damage, intraocular pressure elevation, glaucoma, retinal hemorrhage, iritis, vitreous prolapse, vitritis, iris damage, $\mathrm{CME}$, retinal detachment, IOL subluxation, corneal damage, and localized exacerbation of endophthalmitis. ${ }^{24-26}$ These complications are infrequent in PCCC procedures when compared with Neodymium:YAG laser capsulotomy., ${ }^{9,27}$ Moreover, PCCC is performed as part of the surgery, whereas Neodymium:YAG laser capsulotomy requires a separate session in addition to the surgery. In our cases, we did not encounter any intra-operative complications during application of PCCC. However, it is important to stress that the PCCC procedure is harder to perform and requires more surgical experience, whereas

Neodymium:YAG laser capsulotomy is easier to perform.

In the F.A.C.T. chart, mild refractive disorders and early cataracts generally cause contrast sensitivity losses at higher spatial frequencies, whereas severe refractive disorders and later cataracts cause contrast sensitivity losses at lower spatial frequencies. More severe vision problems such as glaucoma, diabetic retinopathy, macular degeneration, optic neuropathies, and amblyopia may cause degradation of the entire contrast sensitivity curve. ${ }^{28-34}$ In our study, contrast sensitivity curves both under photopic and mesopic conditions did not show any pathological patterns. Although it was statistically insignificant, at the highest spatial frequency $(18 \mathrm{cpd})$ the difference in contrast sensitivity scores was higher than that of other frequencies. This might be due to subclinical PCO in some of the patients.

In conclusion, in the early post-operative period, the PCCC procedure has some positive effects on contrast sensitivity although these effects are statistically insignificant.

\section{Summary}

What was known before

- In the late post-operative period, the PCCC procedure increases the contrast sensitivity when compared with the non-PCCC eyes.

What this study adds

- In the early post-operative period, the PCCC procedure has some positive effects on contrast sensitivity although these effects are statistically insignificant.

\section{Conflict of interest}

The authors declare no conflict of interest.

\section{References}

1 Haegerstrom-Portnoy G, Scchneck ME, Lott LA, Brabyn JA. The relation between visual acuity and other spatial vision measures. Optom Vis Sci 2000; 77: 653-662.

2 Smith AF. Criteria for cataract surgery: the role of visual acuity and visual function. Br J Ophthalmol 1999; 83: 510-511.

3 Pesudovs K, Hazel CA, Doran RML, Elliott DB. The usefulness of Vistech and FACT contrast sensitivity charts for cataract and refractive surgery outcomes research. Br J Ophthalmol 2004; 88: 11-16.

4 Superstein R, Boyaner D, Overbury O. Functional complaints, visual acuity, spatial contrast sensitivity and glare disability in preoperative and postoperative cataract patients. J Cataract Refract Surg 1999; 25: 575-581.

5 Anderson S, Holliday I. Night driving: effects of glare from vehicle headlights on motion perception. Ophthalmic Physiol Opt 1995; 14: 545-551.

6 Frezotti RA, Caporossi A. Pathogenesis of posterior capsule opacification Part I. Epidemiological and clinicostatistical data. J Cataract Refract Surg 1990; 16: 347-352.

7 Ohadi C, Moreira H, McDonnell PJ. Posterior capsule opacification. Curr Opin Opthalmol 1991; 2: 46-52.

8 Solomon KD, Legler UFC, Kostick AMP. Capsular opacification after cataract surgery. Curr Opin Opthalmol 1992; 3: 46-51.

9 Galand A, Cauwenberge F, Moosavi J. Posterior capsulorhexis in adult eyes with intact and clear capsules. J Cataract Refract Surg 1996; 22: 458-461. 
10 Vasavada AR, Desai J. Primary posterior capsulorhexis with or without anterior vitrectomy in congenial cataract. J Cataract Refract Surg 1997; 23: 645-651.

11 Ginsburg AP. Contrast sensitivity and functional vision. Int Ophthalmol Clin 2003; 43: 5-15.

12 Gimbel HV, DeBroff BM. Posterior capsulorhexis with optic capture: maintaining a clear visual axis after pediatric cataract surgery. J Cataract Refract Surg 1994; 20: 658-664.

13 Raina UK, Mehta DK, Monga S, Arora R. Functional outcomes of acrylic intraocular lenses in paediatric cataract surgery. J Cataract Refract Surg 2004; 30: 1082-1091.

14 Schaumberg DA, Dana MR, Christen WG, Glynn RJ. A systematic overview of the incidence of posterior capsule opacification. Ophthalmology 1998; 105: 1213-1221.

15 Buehl W, Sacu S, Findl O. Association between intensity of posterior capsule opacification and contrast sensitivity. Am J Ophthalmol 2005; 140: 927-930.

16 Hayashi K, Hayashi H, Nakao F, Hayashi F. Correlation between posterior capsule opacification and visual function before and after neodymium:YAG laser posterior capsulotomy. AmJ Ophthalmol 2003; 136: 720-726.

17 Jose RMJ, Bender LE, Boyce JF, Heatley C. Correlation between the measurement of posterior capsule opacification severity and visual function testing. J Cataract Refract Surg 2005; 31: 534-542.

18 Barman SA, Hollick EJ, Boyce JF, Spalton DJ, Uyyanonvara $\mathrm{B}$, Sanquinetti G et al. Quantification of posterior capsular opacification in digital images after cataract surgery. Invest Ophthalmol Vis Sci 2000; 41: 3882-3892.

19 Magno BV, Datiles MB, Lasa MSM, Fajardo MRQ, Caruso RC, Kaiser-Kupfer MI. Evaluation of visual function following neodymium:YAG laser capsulotomy. Ophthalmology 1997; 104: 1287-1293.

20 Claesson M, Klaren L, Beckman C, Sjorstrand J. Glare and contrast sensitivity before and after Nd:YAG laser capsulotomy. Acta Ophthalmol 1994; 72: 27-32.

21 Tan JCH, Spalton DJ, Arden GB. The effect of neodymium:YAG capsulotomy on contrast sensitivity and evaluation of methods for its assessment. Ophthalmology 1999; 106: 703-709.

22 Vock L, Menapace R, Stifter E, Bühl W, Georgopoulos M. Effect of primary posterior continuous curvilinear capsulorhexis on clinical performance of ACR6D SE single-piece hydrophilic acrylic intraocular lenses. J Cataract Refract Surg 2007; 33: 628-634.

23 Mela EK, Koliopoulos JX, Pharmakakis NM, Gartaganis SP. Contrast sensitivity after extracapsular and intracapsular cataract extraction. Documenta Ophthalmologica 1998; 95: 121-131.

24 Chambless WS. Neodymium:YAG laser posterior capsulotomy results and complications. Am Intra-Ocular Implant Soc J 1985; 11: 31-32.

25 Steinert RF, Puliafito CA, Kumar SR, Dudak SD, Patel S. Cystoid macular edema, retinal detachment, and glaucoma after Nd:YAG laser posterior capsulotomy. Am J Ophthalmol 1991; 112: 373-380.

26 Billotte C, Berdeaux G. Adverse clinical consequences of neodymium:YAG laser treatment of posterior capsule opacification. J Cataract Refract Surg 2004; 30: 2064-2071.

27 Dholakia SA, Vasavada AR, Nihalani B. Completion rate of primary posterior continuous curvilinear capsulorhexis and vitreous disturbance during congenital cataract surgery. J AAPOS 2006; 10: 351-356.

28 Krasnov MM, Avetisov SE, Makashove NV, Mamikonian VR. The effect of radial keratotomy on contrast sensitivity. Am J Ophthalmol 1988; 105: 651-654.

29 Ginsburg AP, Osher RP, Blauvelt K, Blosser E. The assessment of contrast and glare sensitivity in patients having cataracts. Invest Ophthalmol Vis Sci 1987; 28: 397.

30 Blanchard DL. Contrast sensitivity: a useful tool in glaucoma. Glaucoma 1988; 10: 151.

31 Loshin DS, White J. Contrast sensitivity: the visual rehabilitation of the patient with macular degeneration. Arch Ophthal 1984; 102: 1303-1306.

32 Trick GL, Burde RN, Gordon MO, Santiago JV, Kilo C. The Relationship between hue discrimination and contrast sensitivity deficits in patients with diabetes mellitus. Ophthalmology 1988; 95: 693-698.

33 Rogers GL, Bremer DL, Lequire LE. The contrast sensitivity function and childhood amblyopia. Am J Ophthalmol 1987; 104: 64-68.

34 Hohberger B, Laemmer R, Adler W, Juenemann AG, Horn FK. Measuring contrast sensitivity in normal subjects with OPTEC 6500: influence of age and glare. Graefes Arch Clin Exp Ophthalmol 2007; 245: 1805-1814. 